\title{
Evaluation of the performance of a new freeze desalination technology
}

\author{
T. Mtombeni $\cdot$ J. P. Maree $\cdot$ C. M. Zvinowanda $\cdot$ \\ J. K. O. Asante · F. S. Oosthuizen · W. J. Louw
}

Received: 10 July 2011/Revised: 20 December 2011/Accepted: 20 March 2012/Published online: 7 February 2013

(C) Islamic Azad University (IAU) 2013

\begin{abstract}
The use of desalination technologies which produce concentrated brines is acutely limited by inadequate waste brine disposal mechanisms such that the brine does not contaminate fresh water resources. The treatment of highly saline brine using freeze desalination technique trade marked as HybridICE ${ }^{\mathrm{TM}}$ technology was investigated at pilot scale. The capacity of the HybridICE ${ }^{\mathrm{TM}}$ process to generate fresh water by freeze desalination of brine was investigated in this study. Brine samples to feed into the HybridICE process unit were prepared in tanks with volume capacities between 1.0 and $10.0 \mathrm{~m}^{3}$ by dissolving common salt into tape water. The effects of refrigerant temperature, initial brine concentration, energy consumption were evaluated in relation to product ice quality. Feed brine samples were processed in batches in a closed system where it was continuously re-circulated to generate product ice and more concentrated residual small volume of brine stream. The quality of ice produced could be turned into potable water it terms of its low total dissolved salts and conductivity. The salt removal, based on the average chloride concentration in the ice samples, was $96 \%$. The energy utilization efficiency amounted to an average of ZAR $10.0 / \mathrm{m}^{3}$ water assuming energy cost of ZAR
\end{abstract}

T. Mtombeni · J. P. Maree · C. M. Zvinowanda ( $₫)$.

J. K. O. Asante

Department of Environmental, Water and Earth Sciences,

Tshwane University of Technology, 175 Nelson Mandela Drive,

Arcadia, Pretoria, South Africa

e-mail: zvinowandacm@tut.ac.za

F. S. Oosthuizen

Aqua-Simon UG, Rabenslücke 40, 24944 Flensburg, Germany

W. J. Louw

Marlow Aquatec (Pty) LTD, 1100 Church Street, Colbyn,

Pretoria, South Africa
0.39/kWh. The HybridICE ${ }^{\mathrm{TM}}$ technology was shown to be a better option than other desalination technologies currently in use, in terms of energy utilization and cleaner byproducts.

Keywords Desalination - Heat exchanger - Heat transfer . Freeze desalination · Brine $\cdot$ Impurity

\section{Introduction}

The use of desalination technologies which produce concentrated brines is acutely limited by inadequate waste brine disposal mechanisms such that the brine does not contaminate fresh water resources (Ahmed et al. 2000; Buckley et al. 1987; Muntinh et al. 2009). There are two major problems currently facing industrial water users, namely, the declining availability of fresh water and the deterioration of the quality of available fresh water (Buckley 2005; Schoeman and Steyn 2000; Raluy et al. 2006). This situation is exacerbated by the increase in the use of recycled water from desalination plants resulting in an increased generation of inorganic brines and concentrates (Lewis et al. 2010; Randall et al. 2011). Mining, power generation, petro-chemical and pulp and paper industries employ desalination processes, like spiral reverse osmosis (SRO) and electro-dialysis reversal (EDR), to recover part of their polluted process-waters for reuse (Buhrmann et al. 1999; Dama-Fakir and Toerien 2009).

At Sasol Secunda (a synthetic fuel company in South Africa), $9 \mathrm{ml} /$ day of mine water $[4,000 \mathrm{mg} / \mathrm{L}$ total dissolved solids (TDS)] is desalinated in an EDR and SRO plant to recover about $66 \%$ as fresh water. A residual $3 \mathrm{ml} /$ day brine stream $(12,000 \mathrm{mg} / \mathrm{L}$ TDS $)$ is generated. Freeze desalination using the new HybridICE ${ }^{\mathrm{TM}}$ technology offers 
a novel method of brine treatment. There are two basic methods available for freeze desalination: suspension freeze crystallization (SFC) and progressive freeze crystallization (PFC) (Aider and de Halleux 2009; Flesland 1995; Miyawaki 2001). Both these processes are based on the same freeze separation principle described by Halde (1980), Lorain et al. (2000) and Shone (1987) that due to the small dimensions of the ice crystal lattice, inclusion of compounds in the crystal lattice is impossible except for fluorhydric acid and ammonia. SFC involves the formation of a suspension of numerous small ice crystals in the mother liquor, which are then filtered off to recover pure ice. In PFC, a large single ice crystal is formed on a cold surface and thawed off to recover pure water (Jusoh et al. 2009; Minato et al. 2001; Shirai et al. 1998). Therefore, there is no solid solution with ice. The HybridICE ${ }^{\mathrm{TM}}$ employs the SFC method to accomplish desalination.

The general interest in freeze crystallization, as opposed to other brine treatment technologies such as distillation and evaporation ponds, emanates from the much lower heat of fusion of ice compared to the heat of evaporation of water. The latent heat of fusion of ice is $333 \mathrm{~kJ} / \mathrm{kg}$ and that of evaporation of water is $2,500 \mathrm{~kJ} / \mathrm{kg}$. Wastewater containing toxic compounds (Ruemekorf 1994), heavy metals (Partyka 1986) and organic compounds (van der Ham 1999), which are difficult to treat by conventional methods, can be treated by freeze desalination. The ice crystals produced are excellent latent-heat-storage material capable of storing cold energy of high density (Egolf and Kauffeld 2005; Pronk et al. 2006). This enables free crystallization processes to be used as both wastewater treatment systems and cold heat storage in one facility, which makes load-shifting possible (Wakisaka et al. 2001). Freeze desalination is favorable when dealing with wastewaters rich in volatile organic compounds, unlike distillation which results in the generation of toxic gases or corrosive steam (Jusoh et al. 2008). Freeze crystallization enables the concentration of approximately $80 \%$ of the dissolved compounds in $25 \%$ of the original brine volume (Maurer et al. 2006). Due to low operating temperatures, low cost materials can be used in building freeze desalination plants minimizing capital costs and corrosion potential (Johnson 1976).

According to Jusoh et al. (2008), SFC has proved to have higher productivity than PFC. The traditional barrier to the success of SFC-based brine treatment technologies included the process complexity emanating from the need to grow the individual ice crystals in a growth tank, by Ostwald ripening, and washing in a wash column using a pressurized fresh water stream (Sanchez et al. 2010; Rahman and Ahmed 2007; Qin et al. 2007; Miyawaki et al. 2005). Wakisaka et al. (2001) cited the complexity of iceconcentrate separation as one of the barriers to the success of SFC.
All freeze desalination methods involve crystallization, separation, surface washing and melting of the ice crystals. The ice crystals are then separated from the mother liquor by gravity drainage, centrifuge, filtering, and wash columns (Mahdavi et al. 2011; Thijssen 1969, 1975). Arulampalam et al. (1981) investigated the impact of different parameters on the purification of ice in continuous column crystallizers using an extractive washing model. Axial diffusion of impurities and mass transfer between the entrained brine and ice-melt surrounding the crystals were identified as the most influential factors. Shwartz and Probstein (1968, 1969) investigated the characteristics and performance of laboratory scale counter-washer slurry separators. An ice production rate well below the maximum achievable was reported. All the aforementioned investigations confirm beyond doubt that surface washing is very effective in removing adhering impurities but fail to address the complexity that emanate from the washing process on a pilot or full scale.

The HybridICE ${ }^{\mathrm{TM}}$ system introduced here has the potential to produce high-purity ice crystals without the use of a fresh water stream to wash the ice crystals. This study is aimed at evaluating the potential of the HybridICE ${ }^{\mathrm{TM}}$ technology in treating brines using synthetic wastewater to establish a fundamental comprehension of the occurring phenomena.

The objective of this study was to demonstrate the capability of the HybridICE technology to recover freshwater from industrial brines. The efficiency was evaluated on the basis of energy consumption and impurity removal. The section reported in this study was carried out from January 2010 to April 2011 at the Department of Environmental, Water and Earth Sciences, Tshwane University of Technology, Pretoria. However, the project is still underway.

\section{Materials and methods}

Synthetic brine samples were prepared by varying the amounts of common salt with tape water in tanks of capacities between 1.0 and $10 \mathrm{~m}^{3}$. The concentration of sodium chloride in the aqueous solutions was in the range of 1-25 g/L. Standard silver nitrate solution was used in argentometric titration to determine chloride in the melted product ice and the residual concentrate.

\section{Equipment}

A pilot plant based on the HybridICE ${ }^{\mathrm{TM}}$ process to recover fresh water from contaminated fluids by freeze desalination designed by Sigrotec GmbH, Patent Numbers PCT/DE2008/ 001194 and Germany 102008038 was used in this study.

The module design of the HybridICE plant used in this study is shown in Fig. 1. 
Fig. 1 Modular block diagram for HybridICE ${ }^{\mathrm{TM}}$ desalination plant

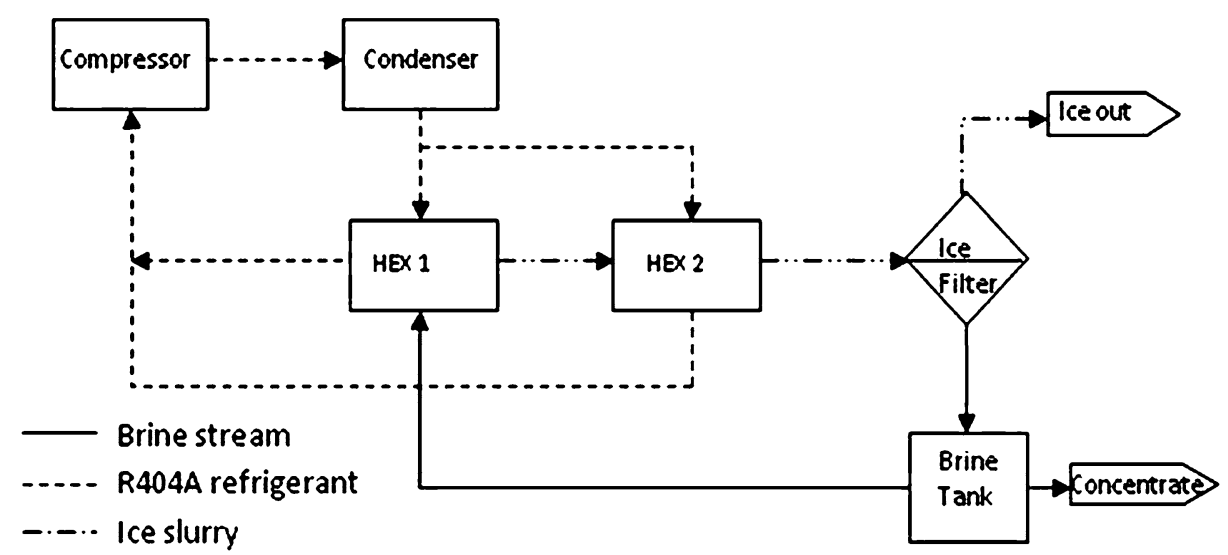

\section{Process description}

The HybridICE unit consists of a refrigeration unit, supplied by Johnson Controls Germany, where a primary refrigerant (R404a) is recycled between the refrigeration unit and the scraped surface heat exchangers (SSHEs). Brine is cooled indirectly to a specific temperature and ice crystals form from pure water molecules in solution. This is accomplished by circulating brine in a closed circuit through the heat exchangers, thereby continuously extracting pure water (as ice) from the concentrate. The SSHEs are configured in series to facilitate nucleation in the first heat exchanger and ripening in the second heat exchanger. Ice crystal growth is achieved by operating the two SSHEs at a temperature differential, the second being at a lower temperature. The first is a counter-current setup while the second is a parallel flow. Slurry is formed and the two phases are separated by an ice filter (Fig. 2).

Slurry of known ice fraction from the SSHEs is delivered to the filter by a centrifugal pump. Upon reaching the much larger space inside the filter, the ice crystals recrystallize, float and agglomerate on the surface of the mother liquor. A growing column of ice (ice cake) then develops due to continuous deposition of ice at the ice/ brine interface. Brine attaches to the ice crystals by interfacial tension. The ice cake becomes drier as it grows as a result of suction induced by the venturi effect in the suction pipe (Fig. 2) as the concentrate circulates coupled with drainage due to gravity as modeled by Henry Darcy (Brown 2002). The processes of re-crystallization, suction and gravity work to defeat the interfacial tension thereby speeding up the drying process. The ice crystal size is therefore a critical parameter in the drying process. The dried ice is then scraped off.

Batches of $1 \mathrm{~m}^{3}$ synthetic sodium chloride brine were processed using the HybridICE ${ }^{\mathrm{TM}}$ pilot plant. Performance of the process was investigated under different operating conditions of pre-set freezing temperature, ice fraction in the slurry, and brine flow-rate through the heat exchangers, with

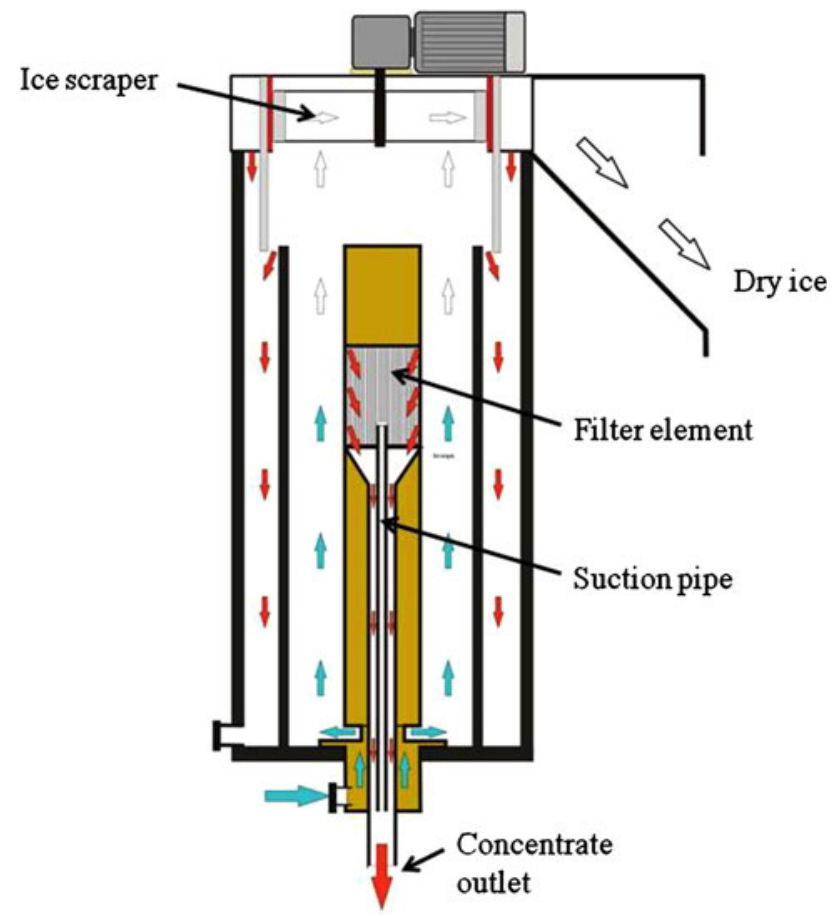

Fig. 2 HybridICE ${ }^{\mathrm{TM}}$ ice-slurry filtering schematic diagram

total recirculation of the concentrate. Freezing temperature, brine flow rate and hence ice fraction were varied and their impact on ice purity and energy consumption was correlated. The performance was determined in terms of impurity removal. The energy consumption for each set of conditions was measured directly by an energy meter. No ice crystals were added as seed ice in the preliminary experiments. Samples of feed brine, the returning brine concentrate and product ice coming out of the filter were collected at their respective outlets at regular intervals for each set of operating parameters. Standard methods for measurement of water quality parameters were used for chloride determination (APHA 1998). Chloride concentration in the product ice, feed, and concentrate were analysed by titrimetric, conductometric and calorimetric (Oosthuizen 2000) methods as a way of 
Table 1 Partitioning of chloride in various streams during desalination with HybridICE process

\begin{tabular}{|c|c|c|c|c|c|c|}
\hline \multirow{2}{*}{$\begin{array}{l}\text { Sample } \\
\text { Parameter }\end{array}$} & \multicolumn{2}{|l|}{ Feed } & \multicolumn{2}{|c|}{ Concentrate from filter } & \multicolumn{2}{|l|}{ Ice } \\
\hline & $\mathrm{Cl}^{-}(\mathrm{mg} / \mathrm{L})$ & Conductivity (mS/cm) & $\mathrm{Cl}^{-}(\mathrm{mg} / \mathrm{L})$ & Conductivity (mS/cm) & $\mathrm{Cl}^{-}(\mathrm{mg} / \mathrm{L})$ & Conductivity $(\mathrm{mS} / \mathrm{cm})$ \\
\hline Run 1 & $10,946.6$ & 26.1 & $17,094.7$ & 33.5 & 699.8 & 1.30 \\
\hline Run 2 & $11,196.5$ & 26.8 & $15,695.1$ & 31.0 & 412.1 & 0.60 \\
\hline Run 3 & $12,246.2$ & 27.7 & $13,945.7$ & 34.3 & 449.7 & 0.80 \\
\hline Run 4 & $14,645.5$ & 35.3 & $23,492.7$ & 43.4 & 450.0 & 0.83 \\
\hline
\end{tabular}

Fig. 3 Effect of brine flow rate and refrigerant temperature on ice fraction

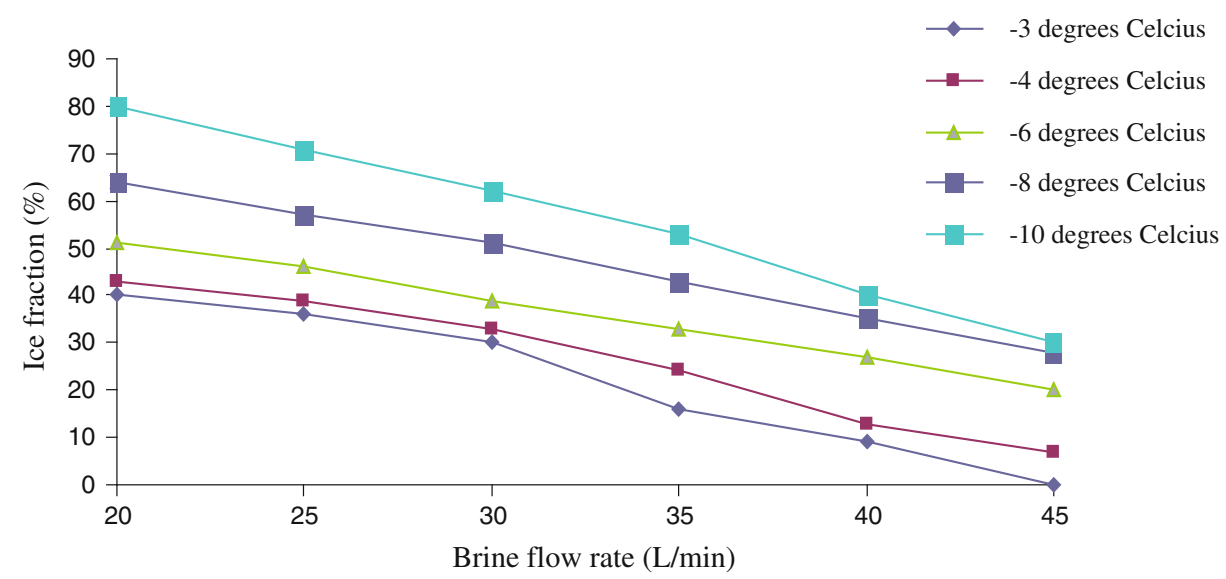

determining the sensitivity and reliability of these analytical procedures. A calibrated WTW Multi340i/SET was used for conductometric tests.

\section{Results and discussion}

The capability of the HybridICE ${ }^{\mathrm{TM}}$ as a freeze desalination technology was measured in terms of impurity removal based on chloride ion concentration in the product ice. The chloride compositions of the simulated brine and the ice samples produced by the HybridICE ${ }^{\mathrm{TM}}$ pilot plant were analysed for chloride content as well as conductivity.

\section{Product quality}

The purity of the ice was measured in terms of the amount of $\mathrm{Cl}^{-}$ions, as well as the electrical conductivity of the melted ice samples. The analysis results are shown in Table 1. It can be observed that the concentration of ionic impurities in the melted ice samples obtained after filtration is very low: $0.09 \%$ $\mathrm{Cl}^{-}$. This shows that the ionic impurities are entrained on the surfaces of the ice crystals by the adhering liquid and not within the crystals. Shirai et al. (1987) investigated incorporation of solutes into agglomerated ice crystals and observed a sharp decrease in impurity concentration with an increase in the number of washings (four washes). Vaessen et al. (2003) washed the ice crystals and obtained the best quality after three washes. The results in Table 1 are also an indication of the effectiveness of the HybridICE ${ }^{\mathrm{TM}}$ filter in removing the excess brine on the ice crystals without washing, which significantly reduces process complexity and operational costs. Khawaji et al. (2008) highlighted the handling of ice and water mixtures as a drawback to freeze desalination. The simplicity of the HybridICE $^{\mathrm{TM}}$ filter system addresses this problem. Moreover, the principle of suction in the HybridICE ${ }^{\mathrm{TM}}$ filter could be employed in eutectic freeze crystallization for further purification of salt crystals instead of washing.

\section{Brine flow rate}

The ice load of the slurry (ice fraction) is predominantly determined by the operating temperature and the brine mass flow. Figure 3 shows the correlation of the three parameters. It can be observed that high ice loads are favored by low temperatures and low flow rates. The ice fraction is depleted as the brine mass flow increases. This phenomenon is a consequence of the variation of brine residence time within the crystallizers and exposure to the driving force of the nucleation process.

Ice fraction

Figure 4 shows the dependence of impurity removal on ice fraction. As the ice load (ice fraction) of the slurry increases, ice purity decreases significantly. The mass fraction of ice in the slurry delivered to the filter determines the rate of deposition of ice at the ice-brine interface inside the filter, hence the ice cake growth rate. At high growth rates, the ice cake 


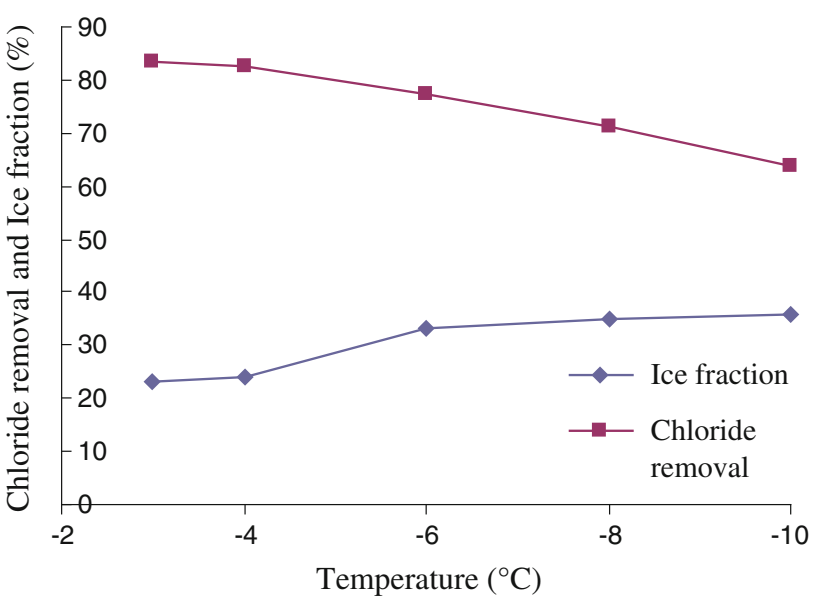

Fig. 4 Effect of refrigerant temperature on chloride removal and ice fraction composition

spends less time exposed to the suction induced by the returning brine, hence the reduction in ice purity. The ice fraction also determines the volume flow-rate of the returning brine available to induce suction in the suction pipe. The suction on the ice cake increases with increasing brine volume flow-rate. Therefore the processes occurring inside the filter are favored by lower ice loads in the slurry.

From Fig. 4, it can also be deduced that ice fraction is a function of the freezing temperature. This is in line with the theory that the temperature difference between the brine and the refrigerant is proportional to the ice yield.

\section{Impurity removal}

The dependence of product ice quality on the initial brine concentration fed into the HybridICE process is shown in Fig. 5. As the brine concentration increased due to continuous freshwater recovery from solution, the inclusion of impurities in the ice crystals remained fairly constant over the running period (Fig. 5). This is an indication of the efficiency of the HybridICE ${ }^{\mathrm{TM}}$ in removing entrained brine from ice crystals.

\section{Energy consumption}

The energy consumption during freeze desalination with the HybridICE process is shown in Table 2. The energy consumption was found to increase with increase in the lowering of freezing point of the brine feed which got more concentrated by each cycle.

Randall et al. (2009) carried out a preliminary cost evaluation of freeze crystallization and evaporative crystallization. The results showed a cost saving of over $80 \%$. The operational cost savings using the HybridICE ${ }^{\mathrm{TM}}$ amounted to $90 \%$. This is attributable to the much lower latent heat of fusion of ice $(333 \mathrm{~kJ} / \mathrm{kg})$, as compared to the latent heat of evaporation of water $(2,500 \mathrm{~kJ} / \mathrm{kg})$. Energy recovery is incorporated in the

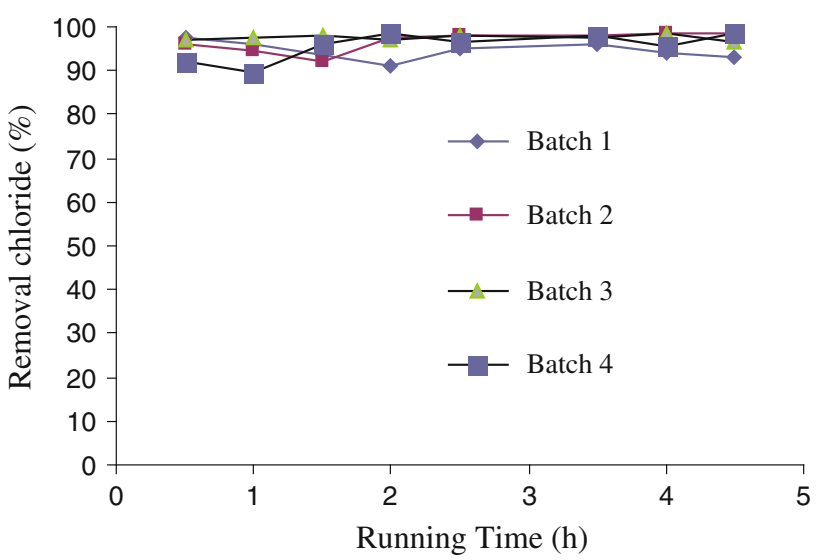

Fig. 5 Chloride removal from feed of different chloride concentrations. Batch 1 Feed $=12 \mathrm{~g} / \mathrm{L}$, Concentrate $=14 \mathrm{~g} / \mathrm{L} ;$ Batch 2 Feed $=11 \mathrm{~g} / \mathrm{L}$, Concentrate $=17 \mathrm{~g} / \mathrm{L} ;$ Batch 3 Feed $=11 \mathrm{~g} / \mathrm{L}$, Concentrate $=14 \mathrm{~g} / \mathrm{L}$; Batch 4 Feed $=15 \mathrm{~g} / \mathrm{L}$, Concentrate $=24 \mathrm{~g} / \mathrm{L}$ )

Table 2 Energy consumption for during freeze desalination

\begin{tabular}{lllll}
\hline Batch & 1 & 2 & 3 & 4 \\
\hline Concentration factor & 1.2 & 1.6 & 1.3 & 1.6 \\
Mass of ice $(\mathrm{kg})$ & 275 & 291 & 334 & 304 \\
Energy $(\mathrm{kWh})$ & 6.5 & 8.4 & 6.9 & 8 \\
Cost $\left(\mathrm{R} / \mathrm{m}^{3}\right)$ & 9 & 11 & 8 & 10 \\
\hline
\end{tabular}

HybridICE $^{\mathrm{TM}}$ system since the brine is circulated continuously through the heat exchangers. This also contributes towards cost savings.

The success of the HybridICE ${ }^{\mathrm{TM}}$ technology depends on the yield of ice as a function of energy input and percentage salt removal. The average salt removal, based on the average chloride analyses from the four runs, was $96 \%$. The salt removal, according to conductivity measurements, averaged $98.5 \%$ and the average energy consumption was $7.45 \mathrm{kWh}$ per tonne of ice produced.

\section{Conclusion}

This study demonstrated the feasibility of treating brine using freeze desalination (by the HybridICE ${ }^{\mathrm{TM}}$ technology). Preliminary results demonstrated the cost effectiveness of this technology in terms of low energy demand, simplicity of process operation and the possibility of generating cold energy from the process.

HybridICE $^{\mathrm{TM}}$ is an environmentally friendly technology and economically feasible, if compared to conventional cooling systems, due to the high energy density of the ice crystals emanating from the latent heat of phase change (melting). HybridICE ${ }^{\mathrm{TM}}$ is preferable when a significant differential exists in electricity tariffs between peak and off-peak rates, in which case load shifting is possible. 
A HybridICE ${ }^{\mathrm{TM}}$ plant can be integrated into an existing refrigeration plant. Retrofitting of existing plants could offset capital costs.

Acknowledgments We thank Tshwane University of Technology, THRIP program of NRF-RSA Grant Number 2010[71807], Rand Water for funding and Aqua-Simon UG for technical assistance.

\section{References}

Ahmed M, Shayya WH, Hoey D, Mahendran A, Morris R, Al-Handaly J (2000) Use of evaporation ponds for brine disposal in desalination plants. Desalination 130:155-168

Aider M, De Halleux D (2009) Cryoconcentration technology in the bio-food industry: principles and applications. Food Sci Technol 42(3):679-685

APHA (1998) Standard methods for the examination of water and wastewaters, 20th edn. ANWA, WEF, Washington

Arulampalam GT, Bates C, Khaw LF, McGrath L (1981) Investigation of purification mechanism in a column crystallizer operating under total reflux and continuous conditions. Desalination 36:87-97

Brown GO (2002) Henry Darcy and the making of a law. Water Resour Res 38(7): 11-1-11-12

Buckley CA (2005) Research into the treatment of inorganic brines and concentrates. Water research commission project no. 201, revision 1.021

Buckley CA, Simpson AE, Kerr CA, Schutte CF (1987) The treatment and disposal of waste brine solutions. Desalination 67:431-438

Buhrmann F, van der Waldt M, Hanekom D, Finlayson F (1999) Treatment of industrial wastewater for reuse. Desalination 124:263-269

Dama-Fakir P and Toerien A (2009) Evaporation rates on brine produced during membrane treatment of mine water. In: International Mine Water Conference, Pretoria, South Africa, 19-23 October 2009

Egolf PW, Kauffeld M (2005) From physical properties of ice slurries to industrial ice slurry applications. Refrigeration 28(1):4-12

Flesland O (1995) Freeze concentration by layer crystallization. Dry Technol 12(89):1713-1739

Halde R (1980) Concentration of impurities by progressive freezing. Water Res 14(6):575-580

Johnson WE (1976) State of the art freezing processes, their potential and future. Desalination 19:349-358

Jusoh M, Yunus RM, Abu Hassan MA (2008) Effect of flow rate and coolant temperature on the efficiency of progressive freeze concentration on simulated wastewater. WASET 47:75-78

Jusoh M, Yunus RM, Abu Hassan MA (2009) Performance investigation on a new design for progressive freeze concentration system. J Appl Sci 9(17):3171-3175

Khawaji AD, Kutubkhanah IK, Wie J (2008) Advances in seawater desalination technologies. Desalination 221:47-69

Lewis AE, Nathoo J, Thomsen K, Kramer HJ, Witkamp GJ, Reddy ST, Randall DG (2010) Design of a eutectic freeze crystallization process for multi-component wastewater stream. Chem Eng Res Des 88(9A):1290-1296

Lorain O, Thiebaud P, Badorc E, Aurelle Y (2000) Potential of freezing in wastewater treatment: soluble pollutant applications. Water Res 35(2):541-547

Mahdavi M, Mahvi AH, Nasseri S, Yunesian M (2011) Application of freezing to the desalination of saline water. Arab J Sci Eng 36:1171-1177

Maurer M, Pronk W, Larsen TA (2006) Treatment processes for sourceseparated urine. Water Res 40(17):3151-3166

Minato W, Shirai Y, Sakashita S (2001) Ice crystallization in a pilot-scale wastewater treatment system. Chem Eng Process 40(3):201-208
Miyawaki O (2001) Analysis and control of ice crystal structure in frozen food and their application to food processing. Food Sci Technol Res 7(1):1-7

Miyawaki O, Liu L, Shirai Y, Sakashita S, Kagitani K (2005) Tubular ice system for scale-up of progressive freeze-concentration. Food Eng 69(1):107-113

Muntinh Y, Mahlaba JS, Pretorius C (2009) Utilising fly ash as a salt sinking media through pasting with industrial brine. In: Proceedings of the congress on engineering (WCE), vol 1, London, July 1-3, pp 1-6

Oosthuizen FS (2000) Application of continuous, calorimetric measuring principle to a technical commercial product: Masters' thesis, Wirtschaftsakademie Schleswig-Holstein Vocational Academy, Faculty of Economic Engineering, Flensburg

Partyka V (1986) Freezing for wastewater recovery. Met Finish 84(11):55-57

Pronk P, Ferreira CAI, Witkamp GJ (2006) Influence of solute type and concentration on ice scaling in fluidized bed ice crystallizers. Chem Eng Sci 61(13):4354-4362

Qin FGF, Chen XD, Premathilaka S, Free K (2007) Experimental study of wash columns used for separating ice from ice-slurry. Desalination 218(1-3):223-228

Rahman MS, Ahmed M (2007) Freezing-melting process and desalination: review of present status and future prospects. Int J Nucl Desalin 2(3):253-262

Raluy G, Serra L, Uche J (2006) Life cycle assessment of MSF, MED and RO desalination technologies. Energy 31:2361-2372

Randall DG, Nathoo J, Lewis AE (2009) Seeding for selective salt recovery during eutectic freeze crystallization. In: Abstracts of the international mine water conference, Pretoria, pp 639-646, 19-23 Oct 2009

Randall DG, Nathoo J, Lewis AE (2011) A case study for treating a reverse osmosis brine using eutectic freeze crystallizationapproaching a zero waste process. Desalination 266(2-3):256-262

Ruemekorf R (1994) Freeze concentration: its application in hazardous wastewater treatment. Environ Sci Pollut Control Ser 7:513-524

Sanchez J, Ruiz Y, Raventos M, Auleda JM, Hernandez E (2010) Progressive freeze concentration for orange juice in a pilot plant falling film. Innov Food Sci Emerg Technol 11:644-651

Schoeman JJ, Steyn A (2000) Investigation into alternative water treatment technologies for the treatment of underground mine water discharged by Grootvlei Proprietary Mines Ltd. into the Blesbokspruit in South Africa. Desalination 133:13-30

Shirai Y, Sugumoto T, Hashimoto M, Nakanishi K, Matsuno R (1987) Mechanisms of ice growth in a batch crystallizer with an external cooler for freeze concentration. Agr Biol Chem Tokyo 51:2359-2366

Shirai Y, Wakisaka M, Miyawaki O, Sakashita S (1998) Conditions of producing an ice layer with high purity for freeze wastewater treatment. Food Eng 38(3):297-308

Shone RDC (1987) The freeze desalination of mine waters. J S Afr Inst Min Metall 87(4):107-112

Shwartz J, Probstein RF (1968) An analysis of counterwashers for freeze distillation desalination. Desalination 4:5-29

Shwartz J, Probstein RF (1969) Experimental study of slurry separators for use in desalination. Desalination 6:239-266

Thijssen HAC (1969) Freeze concentration of food liquids. Food Manuf 44(7):49-54

Thijssen HAC (1975) Apparatus for separation and treatment of solid particles from a liquid suspension. US patent 3,872,009

Vaessen RCJ, Janse BJH, Seckler MM, Witkamp GJ (2003) Evaluation of the performance of a newly developed eutectic freeze crystallizer; scraped cooled wall crystallizer. Inst Chem Eng Trans IchemE Part A 81:1363-1372

Van Der Ham F (1999) Eutectic freeze crystallization. PhD Thesis, Deft University of Technology, The Netherlands

Wakisaka M, Shirai Y, Sakashita, S (2001) Ice crystallization in a pilot-scale freeze wastewater treatment system. Chem Eng Process 40:201-208 